\title{
Optimal Selection of $\rho$-Cycle for Optical Networks. An approach based on Genetic Algorithm
}

\author{
Carlos Colmán ${ }^{1}$ \\ ${ }^{1}$ Universidad Nacional de Asunción, Facultad de Ingeniería \\ San Lorenzo, Paraguay \\ ccolman@ing.una.py \\ Diego Pinto ${ }^{2}$ \\ ${ }^{2}$ Universidad Nacional de Asunción, Facultad de Politécnica \\ San Lorenzo, Paraguay, P.O. Box $N^{o} 2111$ \\ dpinto@pol.una.py \\ and \\ Benjamín Barán ${ }^{1,2}$ \\ Universidad Nacional de Asunción \\ San Lorenzo, Paraguay \\ bbaran@pol.una.py
}

\begin{abstract}
The study of prevention and protection against failures is a central problem in Optical Networks due to the growth of capacity demand for data transmission, especially with the Internet traffic. Without protection, failures of links or nodes cause huge losses of data. The $\rho$ Cycle protection is a novel approach based on pre-configured protection cycles to provide a fast recovery for single link failure. This work proposes a Genetic Algorithm to solve the $\rho$ Cycle protection question. Experimental tests carried out on different topologies have shown that this new proposal is a promising approach to achieve better results when compared to a well-known state-of-the-art alternative called Capacitated Iterative Design Algorithm - CIDA.
\end{abstract}

Keywords: Optical Network, $\rho$-Cycle, Genetic Algorithm.

\section{INTRODUCTION}

The expansion of the Internet makes optical transmission based on wavelength division multiplexing (WDM) a potential successor of today's networks using SONET and SDH technologies, considering that WDM offers higher data transmission rates with better capabilities [1].

Optical transmission networks are also prone to failures caused by various reasons, including cuts in ducts and/or wires, as well as failures in communication equipment. Often, these events have catastrophic consequences for the huge traffic passing through those optical networks [2]. Consequently, the study of tolerance at all stages of optical networks is strategically important.

Among the new and promising strategies for optical protection $[3,4,5]$, the pre-configured protection cycle ( $\rho$ Cycle) seems a promising approach, considering that the design of algorithms that optimize a set of optimal cycles is a crucial task to assure optical network availability. Several articles have addressed the $\rho$-Cycle problem proposing solutions based on different heuristic with unitary population. Thus, this paper intends to apply a new Genetic Algorithm (GA) to solve the problem. Many experimental tests were conducted on various types and sizes of network topologies to prove that the proposed approach improves the performance of a state of the art algorithm: the Capacitated Iterative Design Algorithm (CIDA) proposed by Doucette et al. [6].

\section{Pre-configured Protection Cycle}

Considering the importance of fault tolerance in WDM Networks, Grover et al. proposed a new method inspired in fast recovery strategies of SONET/SDH ringed networks, called $\rho$-Cycle (Pre-configured Protection Cycle) [5]. The 
$\rho$-Cycle approach is a method based on dedicated and pre-calculated protection of links. It was designed to protect the working capacity of each link, reserving up to $50 \%$ of the total link capacity. For example, if the capacity of a WDM Network is 10 wavelength channels, up to 5 wavelength channels may be dedicated to protection. Essentially, the method provides the protection of a unit of working capacity ( $u w c$ ) for links in a cycle, and two units of working capacity $(u w c)$ when a link is surrounded by the cycle (straddling-link) [7]. In Figure 1, an example shows a network before any failure occurs (a), and immediately after a failure (b, c), showing how a $\rho$-Cycle protection provides restoration.

The selection of optimal cycles is a NP-complete problem. Therefore, earlier works used a combination of heuristic algorithms, splitting the problem into two stages:

- $\quad$ First, generating a set of candidates cycles [5 - 11], and later

- $\quad$ selecting a subset of cycles to protect the network [5, 7, and 11].

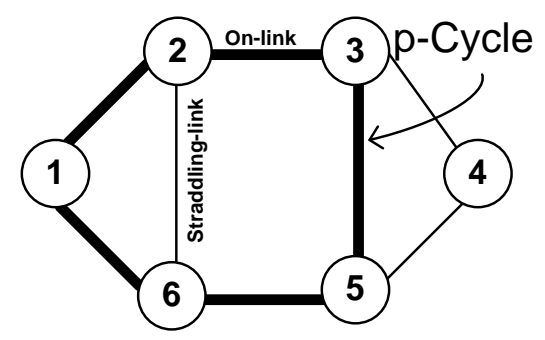

a)

Figure 1: Pre-configured protection $\rho$-Cycle. a) An example of $\rho$-Cycle for a network. b) Protection of one $u w c$ for an on-link. c) Protection of two $u w c$ for a straddling-link. Note that in Figure (b) the protection offers only one path of recovery, while in (c) the protection allows two alternative paths.

To generate candidate cycles, some works suggest heuristics for a topology of $N$ links, getting up to $N^{N}$ candidate cycles [11 - 13]. The complexity of the algorithm to generate cycles proposed in [12] was later studied in [8], proposing an algorithm called SLA (Straddling Link Algorithm), which generates only $N$ candidate cycles. In addition, three algorithms - ADD, EXPAND and GROW - were also proposed as complements of SLA, achieving a substantial improvement in the quality of selected candidates [8].

As elements of assessment and screening, [7] proposed: topological protection (or topological score - TS), efficiency $(\eta)$, and redundancy, in order to improve the selection of optimal cycles. To calculate a solution, an integer linear programming (ILP) formulation was proposed in [5], which is unpractical for large size network topologies due to its complexity. In [6], the Capacitated Iterative Design Algorithms (CIDA) was proposed, achieving a good performance when it works with cycle generators as SLA and GROW. Therefore, this work also uses SLA and GROW as cycle generator algorithms, this time with a Genetic Algorithm to calculate a cyclical protection. This novel approach is called Genetic $\rho$-Cycle $(\mathrm{G} \rho \mathrm{C})$.

\section{Problem Statement}

Let be $\boldsymbol{G}=(V, E)$ a graph representing a topology, $V$ a set of optical nodes and $E$ a set of optical links. For the sake of completeness, the following nomenclature and basic symbols used in this article are defined:

|.| Indicate cardinality of a set.

$v \in V:$ Optical Node.

$e \in E$ : Optical Link.

$\rho \subset E: \quad$ Cycle conformed by a set of links, i.e. $\rho=\left\{e_{1}, e_{2} \ldots, e_{|p|}\right\}$.

$P: \quad$ Set of candidate cycles, i.e. $P=\left\{\rho_{1}, \rho_{2} \ldots, \rho_{P \mid}\right\}$.

$\rho C$ : Set of cycles or $\rho$-Cycle representing a solution, i.e. $\rho C=\left\{\rho_{l}, \rho_{2} \ldots, \rho_{\rho C}\right\}$, where $\rho C \subset P$. 
$X_{\rho}^{e}: \quad$ Ternary variable which indicates the type of protection provided in $e$ for cycle $\rho$.

$$
X_{\rho}^{e}=\left\{\begin{array}{l}
2 \text { if } \text { e is a straddling - link in } \rho \\
1 \text { if } \text { e is an on - link in } \rho \\
0 \text { otherwise }
\end{array}\right.
$$

$Z_{\rho}^{e}: \quad$ Binary variable indicating whether $\rho$ provides protection in $e$.

$$
Z_{\rho}^{e}=\left\{\begin{array}{l}
1 \text { if } \text { e is an on - link or straddling - link } \\
0 \text { otherwise }
\end{array}\right.
$$

Considering the above notation, the problem is to calculate a set of cycle $\rho \mathrm{C} \subset \mathrm{P}$ that maximizes protection efficiency [7]:

where:

$$
\eta_{\rho C}=\frac{T P_{\rho C}}{T C_{\rho C}}
$$

- $\quad$ total protection $T P$ of a set $\rho C$ is defined as:

$$
T P_{\rho C}=\sum_{\rho \in \rho C} T S_{\rho}
$$

- total cost $T C$ of a set $\rho C$ is defined as:

$$
T C_{\rho C}=\sum_{\rho \in \rho C} C_{\rho}
$$

- topological score TS of a cycle $\rho$ is defined in [7] as:

$$
T S_{\rho}=\sum_{e \in E} X_{\rho}^{e}
$$

- $\quad \operatorname{cost} C$ of a cycle $\rho$ is defined in [7] as:

$$
C_{\rho}=\sum_{e \in E} Z_{\rho}^{e}
$$

subject to the full protection constraint of graph $\boldsymbol{G}$ :

$$
\sum_{e \in E} \sum_{\rho \in P^{\prime}} Z_{\rho}^{e} \geq|E|
$$

As a practical example, consider a topology $\boldsymbol{G}$ and a complete set of candidate cycles $P=\left\{\rho_{l}, \rho_{2}, \ldots, \rho_{8}\right\}$ shown in Figure 2.a. Figure 2.b presents a sub-set of cycles $P^{\prime}=\left\{\rho_{4}, \rho_{8}\right\}$ which represents a possible solution of the protection problem; (as described in Figure 2.c where we see that all links are protected). In particular, links $(2,6),(1,3)$ and $(3,5)$ are straddling-links. Figure 2.d shows numerical calculations related to this solution. Note that all links of the given graph are protected fulfilling restrictions (6).

\section{Generator of Candidate CyCles}

For large network topologies, calculating a set of full candidate cycles $P_{\text {full }}$ is unpractical [14]. Several works have proposed a careful construction of a set of non-full candidate cycles $P \subset P_{\text {full }}[9,11]$. SLA and GROW algorithms generate a lot of candidate cycles [14]. Both approaches calculate a set of efficient and adaptable candidate cycles for topologies of different sizes and densities [6, 9, and 14]. Basically, SLA performs two calls to the Dijkstra Shortest Path algorithm to build a cycle for each link. This way, a maximum number of equivalent cycles is generated for the given topology. This will build a reduced, scalable, and efficient set of candidate cycles [8]. 


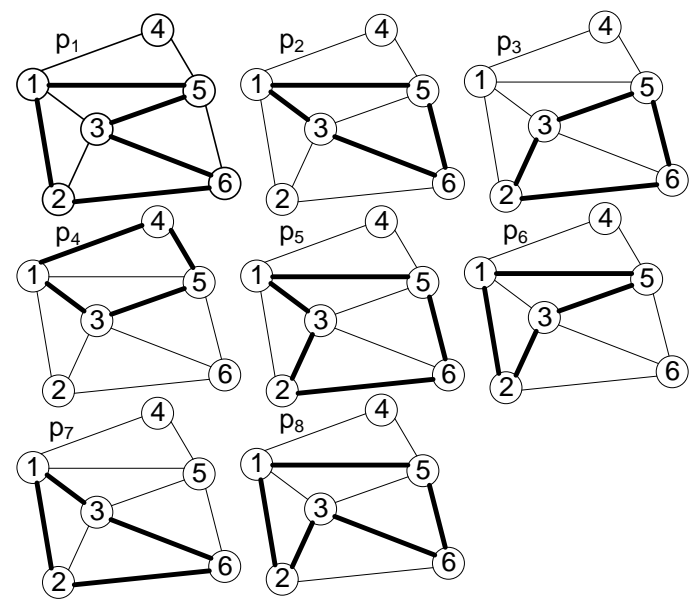

a) Set of candidate cycles

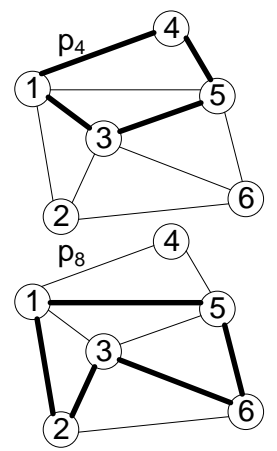

b) A solution $\rho C$

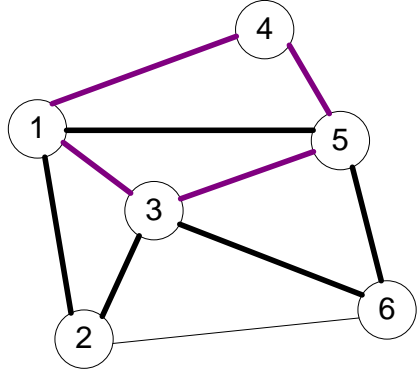

c) Protected Topology

\begin{tabular}{|c|c|c|c|}
\hline$\rho C$ & $\begin{array}{l}\text { Used } \\
\text { Links }\end{array}$ & $\begin{array}{l}\text { Protected } \\
\text { Links }\end{array}$ & $\begin{array}{c}\text { Type of } \\
\text { Protection }\end{array}$ \\
\hline \multirow{5}{*}{$\rho_{4}$} & $(1,4)$ & $(1,4)$ & on $(1 u w c)$ \\
\hline & $(1,3)$ & $(1,3)$ & on $(1 u w c)$ \\
\hline & $(4,5)$ & $(4,5)$ & on $(1 u w c)$ \\
\hline & $(3,5)$ & $(3,5)$ & on $(1 u w c)$ \\
\hline & & $(1,5)$ & $\begin{array}{c}\text { straddling } \\
(2 u w c)\end{array}$ \\
\hline \multicolumn{2}{|c|}{$C_{\rho 4}=4$} & $T S_{\rho 4}=4+2=6$ & \\
\hline \multirow{8}{*}{$\rho_{9}$} & $(1,5)$ & $(1,5)$ & on $(1 u w c)$ \\
\hline & $(5,6)$ & $(5,6)$ & on $(1 u w c)$ \\
\hline & $(6,3)$ & $(6,3)$ & on $(1 u w c)$ \\
\hline & $(3,2)$ & $(3,2)$ & on $(1 u w c)$ \\
\hline & $(1,2)$ & $(1,2)$ & on $(1 u w c)$ \\
\hline & & $(2,6)$ & straddling \\
\hline & & $(1,3)$ & straddling \\
\hline & & $(3,5)$ & $\begin{array}{l}\text { straddling } \\
(2 u w c)\end{array}$ \\
\hline \multicolumn{2}{|c|}{$C_{\rho 9}=5$} & $T S_{\rho 9}=5+6=11$ & \\
\hline
\end{tabular}

d) Evaluation of Objective Function

Figure 2: Example of a protection based on $\rho$-Cycle.

The GROW algorithm implemented in this work is a full version as proposed in [14]. GROW builds new cycles from a set of cycles calculated in advance by another algorithm. In fact, the state-of-the-art proposes a combination of SLA and GROW algorithms to get a refined set of candidate cycles [6]. For example, a first set of cycles is generated using SLA algorithm; next, a second improved set of cycles is obtained from the first one applying GROW algorithm.

\section{CAPACitated ITERATIVE Design Algorithm}

The Capacitated Iterative Design Algorithm (CIDA) is a heuristic tool proposed in [6], which receives a set of candidate cycles $P$. These candidate cycles can be calculated by other algorithms as: SLA and GROW. CIDA calculates the protection efficiency (see equation (1)) of each candidate cycle of $\mathrm{P}$ and then it selects the cycle with the best protection efficiency. This process is repeated until the working capacity is fully protected. The excellent performance of CIDA algorithm together with SLA and GROW approaches are studied in [6, 14] and compared to other techniques such as ILP. Experimental results have demonstrated the potential of CIDA algorithm [9, 11], which is presented below as Algorithm 1.

\section{GENETIC $\rho$-CYCLE APPROACH}

Genetic Algorithm (GA) is a heuristic inspired in Darwing theory of the species evolution and applied to solve complex problems [15]. This work considers an elitist GA, which receives a topology and a set of candidate cycles $\mathrm{P}$ as an input to calculate a set of cycle $\rho C \subset \mathrm{P}$, i.e. a $\rho$-Cycle protection. Then, the best individual $\ell$ of the current population survives unchanged directly to the next evolutionary cycle. This work uses a low elitist pressure, i.e. $\ell=1$. The Genetic Algorithm adapted to solve the $\rho$-Cycle problem is called Genetic $\rho$-Cycle $(\mathrm{G} \rho \mathrm{C})$, which may be considered as the main contribution of this work. Algorithm 2 presents the novel G $\rho \mathrm{C}$ approach which is briefly explained below. 


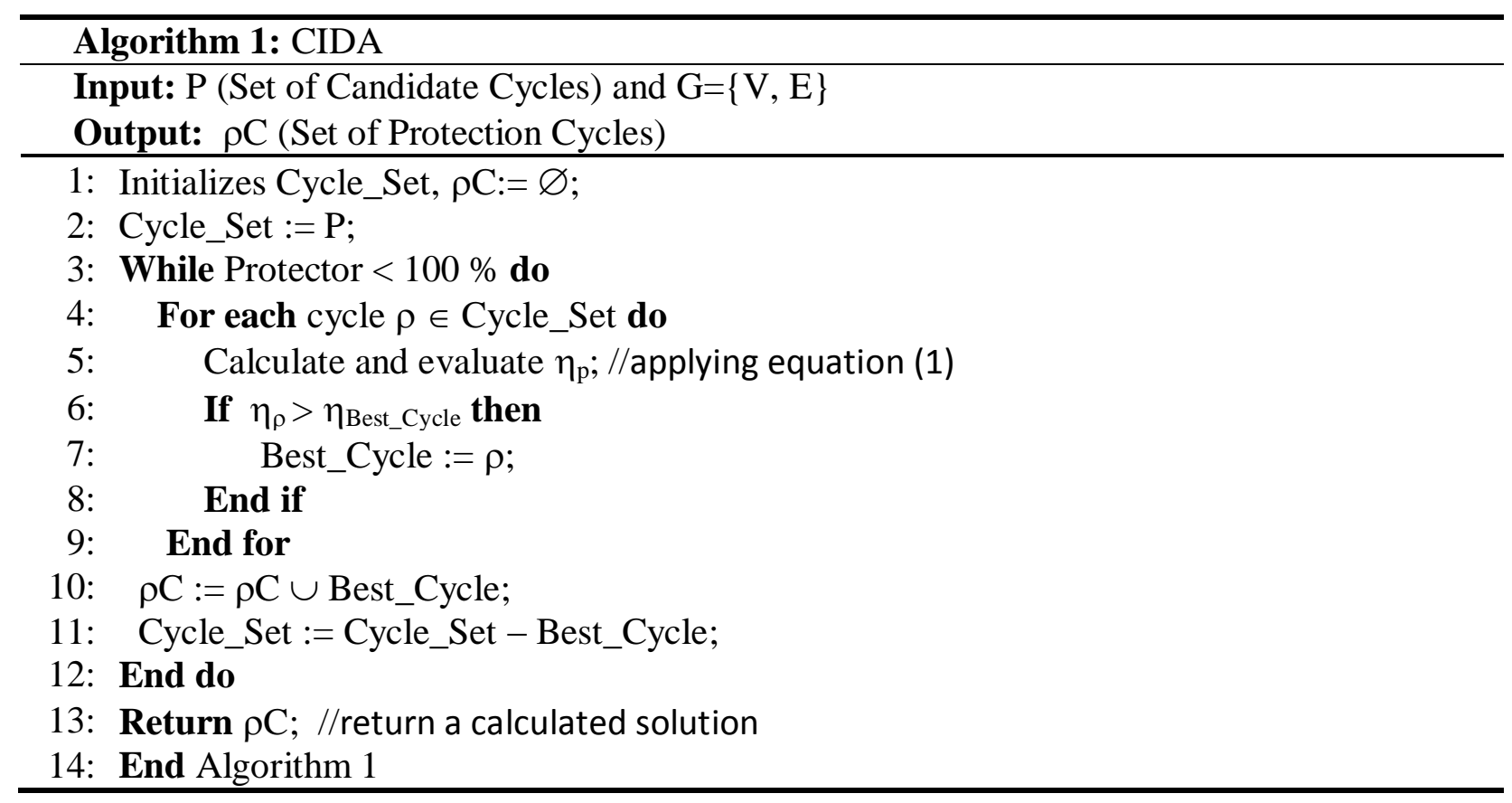

Protection Table. Let us consider a set of candidate cycles $P=\left\{\rho_{1}, \rho_{2}, \ldots, \rho_{|P|}\right\}$. The protection table is a store of cycles $\rho \subset \mathrm{P}$. Each chromosome represents a solution $\rho C \subset P$ which consists of two fields:

- CantCycle: the number of cycles of the solution $\rho \mathrm{C}$, and

- IDs $\rho$-Cycles: index to a table, from which the protection cycles are selected.

Note that the size of a chromosome is dynamic. Figure 3 presents the relationship among protection table, chromosome, and a $\rho \mathrm{C}$ solution considering the example given in Section 3. The structure of a chromosome adopted for this work is similar to a previous approach [22].

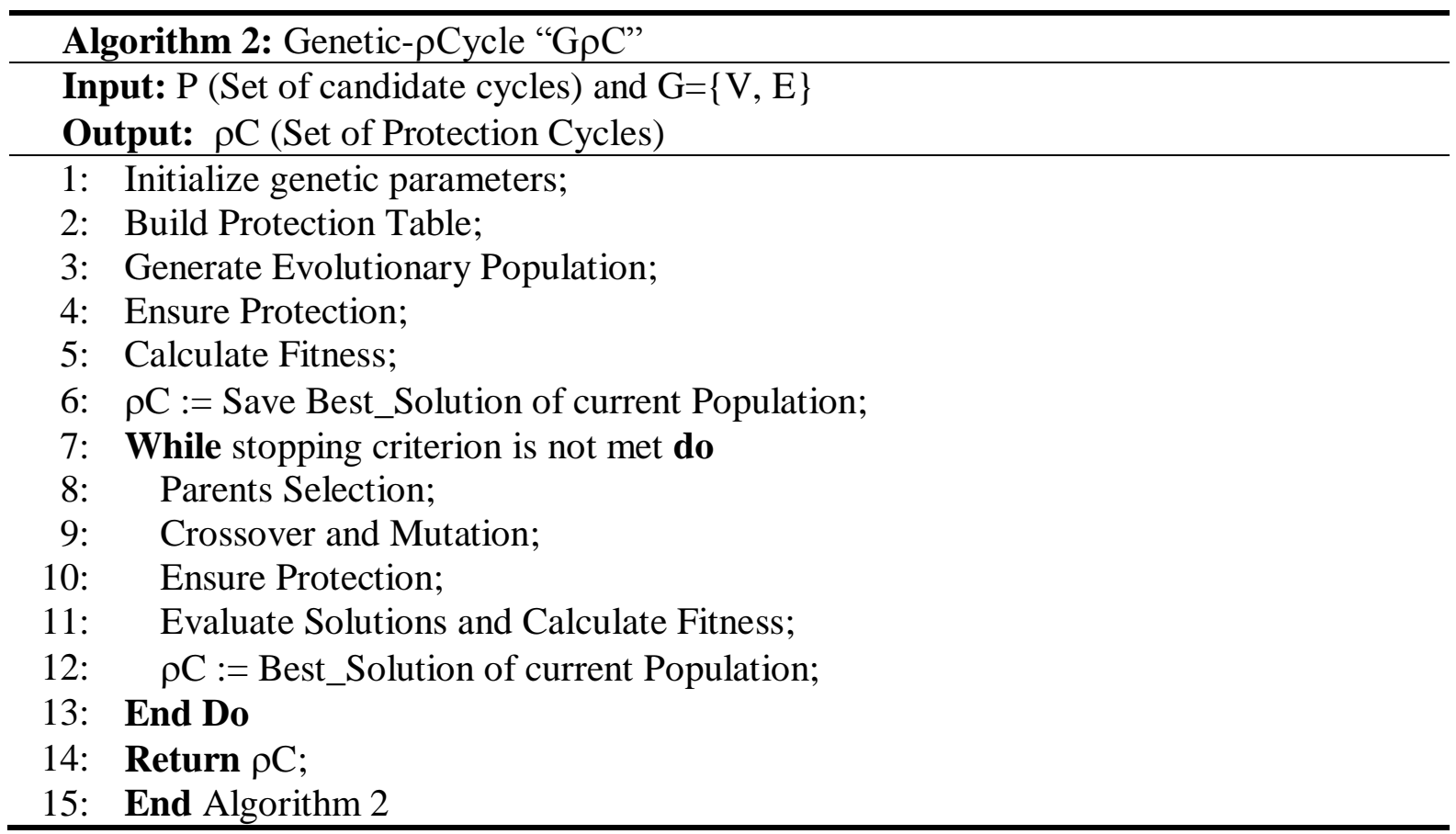

Generate Evolutionary Population. Each chromosome is randomly constructed. Initially, a positive integer number is randomly assigned to CantCycle, CantCycle $\in\{1,2 \ldots,|E|\}$. CantCycle defines the number of genes or cycles to be used. A gene is an index ID to a protection table indicating which cycle will be used. Each gene is randomly 
initialized. To maintain consistency, the following restriction is imposed on genes: gene $_{i} \neq$ gene $_{j}$ where $i \neq j$, i.e. two genes cannot point to a same position in the protection table.

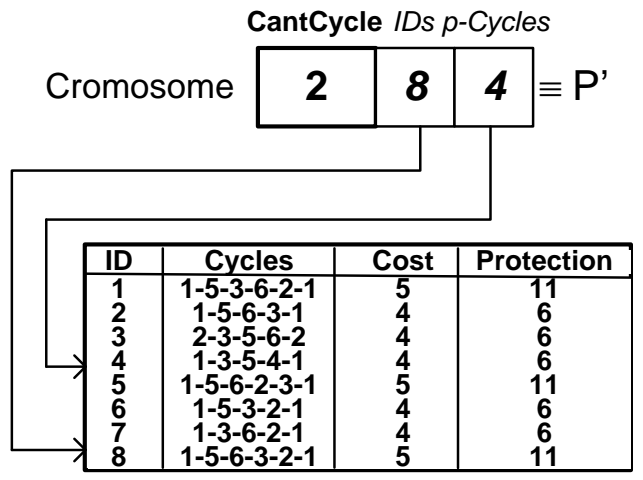

Protection Table $\equiv \mathrm{P}$

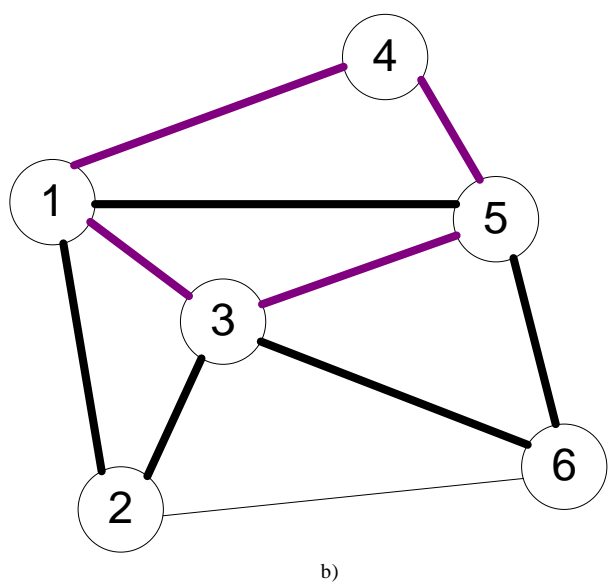

Figure 3: Relationship among chromosome, protection table and solution. Note that a) CantCycle $=2$ indicates the number of cycles of $\rho C$. The following numbers indicate the protection cycles $\rho \in \rho C$. b) the solution considering the chromosome given as example.

Ensure Protection. The solution proposed by a chromosome must verify the constraint of full protection for the whole graph, according to equation (5). Therefore, it adds new different cycles to fulfill the desired protection when some links are not protected.

Calculate Fitness. Efficiency (eq. (1)), considering all cycles of $\rho \mathrm{C}$, is defined as fitness of the chromosome.

Genetic Operators. This work implements a binary tournament as a method for the selection of parents [15]. The adopted crossover operator takes two parents to create a new offspring. This operator has two stages: first, CantCycle is chosen. Then, the known operator BLX [15] is used. The following empty genes are completed randomly with common genes from the two parents. Finally, the mutation involves the replacement individuals, considering a probability of mutation $\mathrm{p}_{\mathrm{m}}=0.2$, experimentally adopted.

\section{EXPERIMENTAL TESTS}

The algorithms were implemented in Mathworks Mathlab 6.5 for Microsoft Windows XP platform. An IBM ThinkPad computer has been used, with Intel Pentium $42.8 \mathrm{MHz}$ processor, $768 \mathrm{MB}$ RAM, and Microsoft Windows XP Professional operating system. For this study, 11 topologies of different sizes and densities were considered, which are presented in Table 1.

The following steps define the experimental process:

- For each topology, 3 sets of candidate cycles were generated. The first set of candidate cycles $\mathrm{P}_{1}$ is generated using SLA, the second set $\mathrm{P}_{2}$ is generated by GROW utilizing $\mathrm{P}_{1}$, and the third set $\mathrm{P}_{3}$ is generated based on $\mathrm{P}_{2}$ using again the GROW algorithm. Considering 3 sets of candidate cycles for 11 topologies, gives a total of 33 sets of candidate cycles, as shown in Table 1.

- 50 independent runs were carried out with G $\rho \mathrm{C}$ and CIDA on each set of candidate cycles, totalizing 3300 runs. A maximum execution time of 20 minutes was used as stopping criterion. Thus, the experiment reported in this work took around 28 days of running time.

Table 2 shows the protection efficiency obtained on different network topologies, while Table 3 summarizes the overall average of experimental results, constructing an overall ranking. Note that $\mathrm{G} \rho \mathrm{C}$ presents a significant superiority in all instances. For low complexity in size networks as ARPA topology, the two approaches (CIDA and $\mathrm{G} \rho \mathrm{C}$ ) reached the same set of optimal protection; however, the advantage of the proposed method in more complex networks is highly noticeable. In fact, it is noteworthy that the efficiency of different $\mathrm{G} \rho \mathrm{C}$ solutions increases with the complexity of candidate cycles, i.e. the efficiency of an optimal solution is directly proportional to the number and quality of candidate cycles. 
Considering the whole set of candidate cycles and overall results of Table 3, it can be seen that CIDA improves $18 \%$ $(=100 *(1.83-1.55) / 1.55)$, while GpC improves $45 \%$ (= $100 *(2.44-1.68) / 1.68)$, obtaining a better improvement. These trends indicate that the efficiency of $\mathrm{G} \rho \mathrm{C}$ increases faster than CIDA with the quality of the candidate cycles, which demonstrates the ability of $\mathrm{G} \rho \mathrm{C}$ to explore huge space solutions.

Table 1: Network Topologies with numbers of nodes, links and candidate cycles generated by SLA and GROW algorithms.

\begin{tabular}{c|l|cc|ccc}
\hline Symbols & \multicolumn{1}{|c|}{ Network Topologies } & $\begin{array}{c}\text { Number of } \\
\text { Nodes }\end{array}$ & $\begin{array}{c}\text { Number of } \\
\text { Links }\end{array}$ & $\begin{array}{c}\text { SLA } \\
\left|\mathrm{P}_{1}\right|\end{array}$ & $\begin{array}{c}\text { GROW } \\
\left|\mathrm{P}_{2}\right|\end{array}$ & $\begin{array}{c}\text { GROW } \\
\left|\mathrm{P}_{3}\right|\end{array}$ \\
\hline NSF & $\begin{array}{l}\text { USA National Science Foundation } \\
\text { Backbone Network [1] }\end{array}$ & 14 & 25 & 25 & 144 & 756 \\
\hline BCBY & Bell Core Backbone Yerse LATA [17] & 15 & 27 & 27 & 122 & 780 \\
\hline ULHN & USA Long Haul Network [6] & 28 & 45 & 45 & 181 & 780 \\
\hline ARPA & USA ARPA Backbone Network II [18] & 21 & 25 & 25 & 30 & 30 \\
\hline BCN & Bell Core Backbone Network [16, 18] & 25 & 28 & 28 & 120 & 404 \\
\hline PAN & PAN European COST 239 [1, 16] & 11 & 26 & 25 & 106 & 366 \\
\hline ELHN & $\begin{array}{l}\text { European Long Haul Network - } \\
\text { France Telecomm [6, 11] }\end{array}$ & 43 & 71 & 70 & 321 & 1683 \\
\hline GOBN & $\begin{array}{l}\text { German Optical Backbone Network } \\
{[19]}\end{array}$ & 17 & 27 & 26 & 79 & 212 \\
\hline ECN & $\begin{array}{l}\text { ECNet European Backbone Network } \\
{[20]}\end{array}$ & 18 & 39 & 25 & 144 & 420 \\
\hline CNBN & $\begin{array}{l}\text { China National Backbone Network } \\
{[21]}\end{array}$ & 66 & 120 & 120 & 662 & 4257 \\
\hline NTT & $\begin{array}{l}\text { Nippon Telephone Telegraph } \\
\text { Backbone Network [22] }\end{array}$ & 49 & 66 & 66 & 213 & 545 \\
\hline
\end{tabular}

Table 2: Experimental Results by Topologies.

\begin{tabular}{|c|c|c|c|c|}
\hline \multirow{2}{*}{ Topology } & \multirow{2}{*}{ Approach } & \multicolumn{3}{|c|}{ Average of Protection Efficiency $(\eta)$} \\
\hline & & $S L A_{P 1}$ & $G_{\text {GROW }}$ & $\mathrm{GROW}_{P 3}$ \\
\hline \multirow{2}{*}{ NSF } & CIDA & 1.48 & 1.73 & 2.57 \\
\hline & $\mathrm{G} \rho \mathrm{C}$ & 1.70 & 2.73 & 2.83 \\
\hline \multirow{2}{*}{ ULHN } & CIDA & 1.5 & 1.8 & 1.8 \\
\hline & $\mathrm{GpC}$ & 1.6 & 2.28 & 2.4 \\
\hline \multirow{2}{*}{$\mathrm{BCN}$} & CIDA & 1.8 & 1.86 & 1.86 \\
\hline & $\mathrm{GpC}$ & 1.8 & 2.52 & 2.87 \\
\hline \multirow{2}{*}{ ELHN } & CIDA & 1.43 & 1.53 & 1.61 \\
\hline & $\mathrm{GpC}$ & 1.60 & 1.97 & 2.05 \\
\hline \multirow{2}{*}{$\mathrm{ECN}$} & CIDA & 1.65 & 1.90 & 1.95 \\
\hline & $\mathrm{GoC}$ & 1.80 & 2.60 & 2.72 \\
\hline \multirow{2}{*}{ NTT } & CIDA & 1.35 & 1.40 & 1.50 \\
\hline & $\mathrm{GpC}$ & 1.41 & 1.60 & 1.73 \\
\hline \multirow{2}{*}{ BCBY } & CIDA & 1.78 & 1.9 & 1.95 \\
\hline & $\mathrm{G} \rho \mathrm{C}$ & 1.95 & 2.36 & 2.56 \\
\hline \multirow{2}{*}{ ARTA } & CIDA & 1.10 & 1.20 & 1.20 \\
\hline & $\mathrm{GpC}$ & 1.10 & 1.20 & 1.20 \\
\hline \multirow{2}{*}{ PAN } & CIDA & 1.81 & 1.82 & 2.03 \\
\hline & $\mathrm{G} \rho \mathrm{C}$ & 2.00 & 2.81 & 3.32 \\
\hline \multirow{2}{*}{ GOBN } & CIDA & 1.53 & 1.93 & 1.96 \\
\hline & $\mathrm{GpC}$ & 1.70 & 2.70 & 2.89 \\
\hline \multirow{2}{*}{ CNBN } & CIDA & 1.60 & 1.62 & 1.70 \\
\hline & $\mathrm{G} \rho \mathrm{C}$ & 1.81 & 2.06 & 2.23 \\
\hline
\end{tabular}


Table 3: Overall Average Efficiency.

\begin{tabular}{c|cc|cc|cc}
\hline \multirow{2}{*}{ Algorithms } & \multicolumn{2}{|c|}{ SLA $_{\mathbf{P 1}}$} & \multicolumn{2}{c|}{ GROW $_{\mathbf{P 2}}$} & \multicolumn{2}{c}{ GROW $_{\mathbf{P 3}}$} \\
\cline { 2 - 7 } & Averages & $\begin{array}{c}\text { Standard } \\
\text { Deviation }\end{array}$ & Averages & $\begin{array}{c}\text { Standard } \\
\text { Deviation }\end{array}$ & Averages & $\begin{array}{c}\text { Standard } \\
\text { Deviation }\end{array}$ \\
\hline CIDA & 1.55 & 0 & 1.70 & 0 & 1.83 & 0 \\
GpC & 1.68 & 0 & 2.26 & 0.03 & 2.44 & 0.03 \\
\hline
\end{tabular}

\section{CONCLUSION AND FUTURE WORK}

This work proposes the use of an elitist Genetic Algorithm to address the problem of protecting networks based on pre-configured protection cycles or $\rho$-Cycle. Following the outline of the state-of-the-art, our approach solves the $\rho$ Cycle problem in two stages, starting with the construction of a set of candidate cycles using two generators of cycles: the SLA and the GROW algorithms. As a second step, a GA optimizer was implemented to calculate a set of optimal protection cycles. Experimental results indicate that the proposed approach $\mathrm{G} \rho \mathrm{C}$ is really promising, obtaining in almost all cases better solutions than those provided by one of the best state-of-the-art algorithms, CIDA.

As future work, the authors propose to design an algorithm which is independent of a cycle list in order to get a full space exploration. In addition, it intends to consider the protection of shared risk groups and the study of cases where traffic is known in advance.

\section{References}

[1] A. K. Somani. Survivality and Traffic Grooming in WDM Optical Networks. Cambridge University Press, 2005.

[2] P.F. Falcao. Pan-European multi-wavelength transport networks. Network: design, architecture, survivability and SDH networking. Proceedings of the 1st International Workshop on Reliable Communication Networks. Brugge, Belgium, (May, 1998).

[3] G. Mohan, C. Siva Ram Murthy and A.K. Somani. Efficient algorithms for routing dependable connections in WDM optical networks. IEEE/ACM Transactions on Networking. Vol 9, No. 5, 2001, pp. 553-566.

[4] M.T. Frederick and A.K. Somani. A single-fault recovery strategy for optical networks using sub-graph routing. 7th IFIP Working Conference on Optical Networks Design and Modeling (ONDM 2003). Budapest, Hungary, (February, 2003).

[5] W. D. Grover and D. Stamatelakis. Cycle-oriented distributed pre-configuration: Ring-like speed with meshlike capacity for self-planning network restoration. Proc. IEEE International Conference on Communications (ICC). Atlanta, Georgia, USA, (Jun, 1998), pp. 537-543.

[6] J. Doucette, D. He, W. D. Grover and O. Yang. Algorithmic approaches for efficient enumeration of candidate $\rho$-Cycles and capacitated $\rho$-Cycle network design. Proc. Fourth International Workshop on the Design of Reliable Communication Networks (DRCN 2003). Banff, Alberta, Canada, (October, 2003), pp. 212-220.

[7] D. A. Schupke, C. G. Gruber and A. Autenrieth. Optimal configuration of r-Cycles in WDM networks. Proc. IEEE International Conference on Communications (ICC). New York City, USA, (April - May, 2002), pp. 2761-2765.

[8] H. Zhang and O. Yang. Finding protection cycles in DWDM networks. Proc. IEEE International Conference on Communications (ICC). Nueva York, USA, (May, 2002), pp. 2756-2760.

[9] T. Zhao, H. Yu and L. Li. A novel algorithm for node-encircling and link candidate $\rho$-Cycles design in WDM mesh network. Journal of the Chinese Institute of Engineers. Vol 29, No. 7, 2006, pp. 1227-1233.

[10]W. D. Grover and J. Doucette. Advances in optical network design with $\rho$-Cycles: Joint optimization and preselection of candidate $\rho$-Cycles. Proc. IEEE LEOS Summer Topicals 2002. Mont Tremblant, Québec, Canada, (July, 2002), pp. 49-50. 
[11]C. Liu and L. Ruan. Finding good candidate cycles for efficient $\rho$-Cycle network design. Proc. 13th International Conference on Computer Communications and Networks. Chicago, Illinois, USA, (October, 2004), pp. 321-326.

[12]D. B. Johnson. "Finding all the elementary circuits of a directed graph". SIAM Journal on Computing, (April, 1975), pp. 77-84.

[13]D. Stamatelakis and W. D. Grover. Network restorability design using pre-configured trees, cycles, and mixtures of pattern types. TRLabs, Edmonton, Alberta, Canada, Technical Report TR-1999-05, 2000.

[14]Farkas, J. Szigeti and T. Cinkler. $\rho$-Cycle Based Protection Scheme for Multi-Domain Networks. 5th International Workshop on Design of Reliable Communication Networks (DRCN). Naples, Italy, (October, 2005).

[15]D. E. Goldberg. Genetic Algorithms in Search, Optimization, and Machine Learning. Addison-Wesley Publishing Company, 1989.

[16]Liu, L. Ruan. $\rho$-Cycle Design in Survivable WDM Networks with Shared Risk Link Groups (SRLGs) (extended version). Photonic Network Communications. Vol 11, Issue 3, (May; 2006), pp. 301 - 311.

[17]W. D. Grover and D. Stamatelakis, Bridging the ring-mesh dichotomy with $\rho$-Cycles. Proc. Second International Workshop on the Design of Reliable Communication Networks (DRCN 2000). Munich, Germany, (April, 2000), pp. 92-104.

[18] Shen and W. D. Grover. Extending the $\rho$-Cycle concept to path segment protection for span and node failure recovery. IEEE Journal on Selected Areas in Communications. Vol 21, No. 8, (October, 2003), pp. 1306-1319.

[19]D. A. Schupke. Automatic Protection Switching for $\rho$-Cycles in WDM Networks. Optical Switching and Networking (OSN). Elsevier, Vol 2, No. 1, (May, 2005), pp. 35-48.

[20]H. Huang and J. A. Copeland. Hamiltonian cycle protection: A novel approach to mesh WDM optical network protection. Proc. IEEE Workshop on High Performance Switching and Routing (HPSR). Dallas, Texas, USA, (May, 2001), pp. 31-35.

[21]Z. Zhang et al. $\rho$-Cycle-based protection design for IP over WDM networks. Proc. Asia-Pacific Optical and Wireless Communications Conference (APOC). Wuhan, China, Vol 5282, (November, 2003), pp. 598-608.

[22]J. Crichigno and B. Barán. Multiobjective Multicast Routing Algorithm for Traffic Engineering. IEEE International Conference on Computers and Communication Networks. Illinois, USA, (October, 2004), pp. 301306 